Tous droits de traduction, d'adaptation et de reproduction par tous procédés, réservés pour tous pays. La loi du 11 mars 1957 n'autorisant, aux termes des alinéas 2 et 3 de l'article 41, d'une part, que les « copies ou reproductions strictement réservées à l'usage privé du copiste et non destinées à une utilisation collective " et, d'autre part, que les analyses et les courtes citations dans un but d'exemple et d'illustrations, « toute représentation ou reproduction intégrale, ou partielle, faite sans le consentement de l'auteur ou de ses ayants droit ou ayants cause, est illicite" (alinéa 1er de l'article 40).

Cette représentation ou reproduction, par quelque procédé que ce soit, constituerait donc une contrefaçon sanctionnée par les articles 425 et suivants du Code pénal.

Armand Colin éditeur. Paris - Dépôt légal : 1996 - No 6737 - No 4, juillet-août 1996 


\section{EDITIONS
EIHE|S|S}

Éditions de l'École des Hautes Études en Sciences Sociales

\section{MARC RAEFF Politique et culture en Russie, 18e-20e siècles}

Les structures sociales et institutionnelles de la Russie moderne, ébauchées par les rudes mains de Pierre le Grand, ont été travaillées par des forces contradictoires. La centralisation administrative et la réglementation bureaucratique, qu'a promues le premier empereur, se sont heurtées à une culture politique d'origine européenne en quête d'un essor social maximal. A contrario, les structures sociales de l'Empire étaient régies par le servage des paysanneries et l'absence de corps organisés disposant de droits et de privilèges garantis par la loi. Le souverain autocrate seul pouvait briser cette impasse ; mais les efforts des monarques modernisateurs des institutions ont justifié et consolidé l'arbitraire.

L'ouverture sur l'Europe travaillait les cultures russes : fenêtre avec Pierre le Grand, large porte à la fin du $18 \mathrm{e}$ siècle, avant une diffusion de masse au début du 20 e siècle. Idées, valeurs, goûts provenant d'Europe occidentale n'étaient d'ailleurs pas uniformes ou homogènes. Le rythme de la diffusion n'était pas constant, et ces ruptures de temporalités en altéraient le dynamisme, contraint en profondeur par les conditions sociales et mentales particulières des élites russes.

De telles contradictions entre les institutions et les cultures de la Russie moderne ont fécondé la littérature et les arts ; elles ont aussi conduit le régime impérial à un cul-de-sac politique. Les révolutions de 1917 ambitionnaient de sortir de celui-ci, en jetant les bases d'un ordre nouveau qui écarterait ces dilemmes structurels et sauverait le dynamisme culturel. Les élites dans l'exil ont continué de leur côté la renaissance culturelle amorcée au début du siècle ; et leurs efforts n'ont pas peu contribué à faire connaître à l'étranger la Russie, ses réussites et ses problèmes.

Les études réunies dans ce recueil veulent rendre compte de ces évolutions et de ces tensions de longue durée, donnant à voir une Russie à la fois «absente et présente " au monde moderne.

Recherches d'histoire et de sciences sociales, 65 1996. 290 pages $-150 \mathrm{~F}$

\section{DIFFUSION \\ Clid}

131 bd Saint-Michel, 75005 Paris

Tél : 43544715 Fax : 43548073 


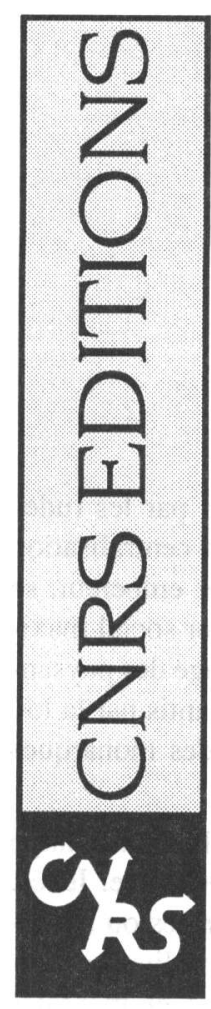

OLYMPIE LA VIITOIRE POUR LES DIEUX

\section{Philippe de CARBONNIERES}

\section{Collection "Patrimoine de la Méditerranée"}

Le prestige d'Olympie est universel, et la renommée de ses Jeux fut telle, depuis toujours, qu'elle a provoqué leur résurrection il y a bientôt un siècle. Le sile lui-même est moins connu que ceux d'Athènes ou de Delphes, encore que sa beauté et sa douceur, la charge d'émolion dont il est empreint, attirent à lui de nombreux visiteurs. Brillomment fouillé et étudiè, c'est surlout sous l'angle archéologique ou celui de l'histoire de l'art qu'il est généralement présenté. Si les pays germaniques ou anglo-saxons ont depuis longtemps compris limportance du sport dans le monde grec, il n'en est pas de même dans notre pays où le sujet n'est qu'effleuré ou limité au plan esthétique. II étail donc nécessaire d'envisager le sanctuaire d̀ travers ses vestiges el ses légendes fondatrices, mois plus encore selon un fil directeur : les Jeux, dans leur déroulement, leurs ospects lechniques, leur impact politique ef cullurel. Lieu de pelerinage, terre de rassemblement por deld les conflits, ce nom magique évoque aussi, pendant toute l'Antiquité, le sommet de la gloire athletique. Ourant au mains douze siècles, Olympie a symbolisé le sport et sa fraternite. Il offre aujourd'hui encore un des plus beaux modèles d'humanité.

$19,5 \times 24-128$ pages

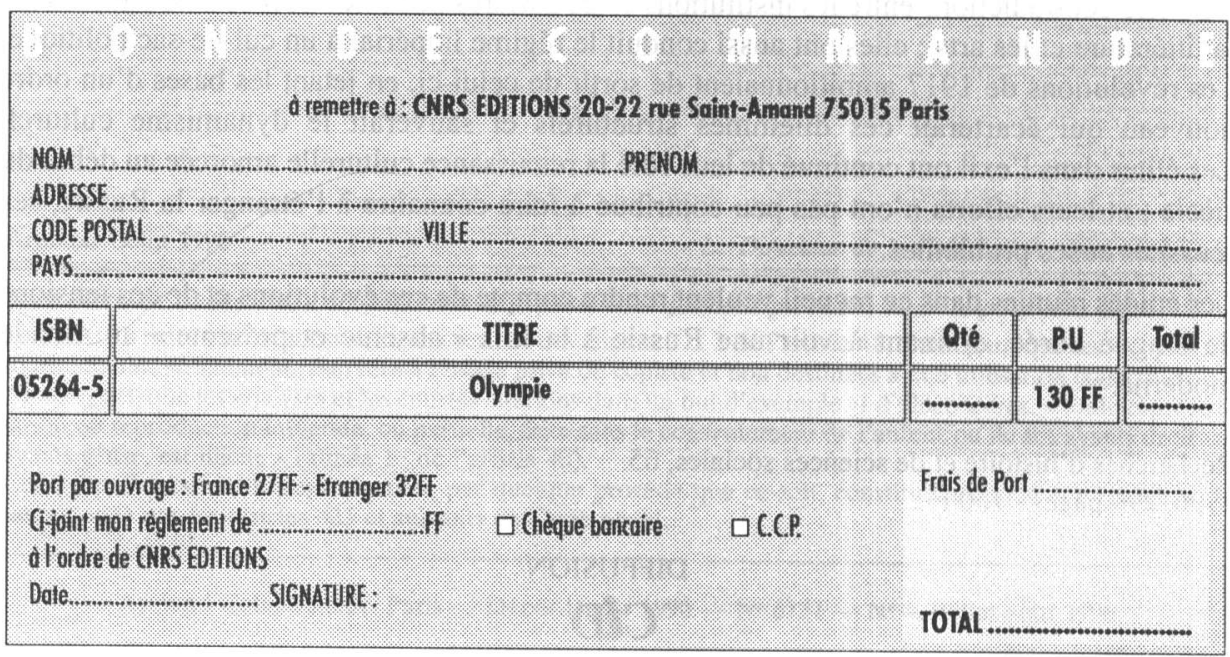


L'INED publie Population, une revue bimestrielle (51e année). Depuis 1989 , un numéro annuel en anglais : Population: An English Selection présente une sélection d'articles parus principalement dans Population.

\section{POPULATION $-n^{\circ} 2-1996$}

Louis RousSEL

José Rose

Alain Blum et

Irina TroITSKAJA

Richard LALOU et

Thomas K. LEGRAND

Rossella Palomba, Luciana QUATTROCIOCCHI

Michèle BiÉGeLmanN-

MASSARI

\section{— Hommage à Alain Girard}

- De quelques usages du nombre en sociologie. Autour des travaux d'Alain Girard, de la formation et de l'insertion des jeunes

- La mortalité en Russie aux XVIII et $\mathrm{XIX}^{\mathrm{e}}$ siècles : estimations locales à partir des Revizii

- La mortalité des enfants du Sahel en ville et au village

- Images de la famille italienne en mutation

- Les dispenses civiles au mariage de 1960 à 1992. II. Le mariage posthume : mariage de raison ou mariage d'amour?

\section{*}

LE CINQUANTIEME ANNIVERSAIRE DE L'INED par Roland Pressat, Michel Loriaux,

Louis Roussel et Jean-Claude Chasteland, Benoît RIANDEY

\begin{tabular}{|lccc|}
\hline \multicolumn{4}{|c|}{ Les demandes d'abonnement sont à adresser à l'INED } \\
France, Dom, Tom & Étranger & Par avion \\
.Le numéro de Population & $85 \mathrm{FF}$ & $100 \mathrm{FF}$ & \\
.Le numéro annuel en Anglais & $180 \mathrm{FF}$ & $195 \mathrm{FF}$ & \\
.Abonnement d'un an à Population & $400 \mathrm{FF}$ & $455 \mathrm{FF}$ & $535 \mathrm{FF}$ \\
.Avec le numéro en anglais & $580 \mathrm{FF}$ & $650 \mathrm{FF}$ & $730 \mathrm{FF}$ \\
Veuillez joindre à votre demande un chèque à l'ordre de l'agent comptable de l'INED \\
ou un virement postal CCP Paris $9061-56 h$.
\end{tabular}

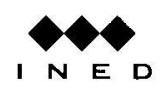

INSTITUT NATIONAL D'ÉTUDES DÉMOGRAPHIQUES

27 rue du Commandeur, 75675 Paris Cedex 14, France Tél. : 33 (1) 421820 00, Fax : 33 (1) 42182199 


\section{Revue française de sociologie}

publiée avec le concours du

CENTRE NATIONAL DE LA RECHERCHE SCIENTIFIQUE

et de L'INSTITUT DE RECHERCHE SUR LES SOCIÉTÉS CONTEMPORAINES

59-61, rue Pouchet, 75849 Paris Cedex 17 - Tél. : 40.25.11.87 ou 88

AVRIL-JUIN 1996, XXXVII-2

ISBN $270800801-3$

\section{Les marchés du travail universitaires, comme économie de la qualité Christine MUSSELIN}

Volontarisme et rationalité d'État.

La politique de la ville Gérard CHEVALIER

Processus différentiels d'intégration au sein des familles algériennes en France

Ahsène ZEHRAOUI

Processus de socialisation et vécu émotionnel des enfants Cléopâtre MONTANDON

La fin des contremaîtres traditionnels? Philippe TROUVÉ

LES LIVRES

Abonnements :

L'ordre et le paiement sont à adresser directement à :

Editions OPHRYS BP 8705003 GAP Cedex - CCP Marseille $63609 \mathrm{E}$

Les abonnements sont annuels et partent du premier numéro de l'année en cours.

Tarif 1996: L'abonnement (4 numéros) France

$360 \mathrm{~F}$

Etranger ................ $440 \mathrm{~F}$

Vente au numéro: $\quad$ Soit par correspondance auprès de :

Editions OPHRYS - Tél. : 92 53.85.72

Soit auprès des librairies scientifiques

Le numéro 


\section{LE DROIT ANTISÉMITE DE VICHY}

Actes du colloque organisé en décembre 1994 par l'université de Bourgogne sous la direction de Dominique Gros.

Pascal Ancel • Claire Andrieu - Robert Badinter Jean-Marc Béraud - Marguerite Blocaille-Boutelet Didier Boden - Pierre Bodineau • Hervé Bonnard Denis Broussolle • Olivier Camy • Jean-Jacques Clère Michel Deguy • Jean-Pierre Dubois • Olivier Duhamel Gérard Fritz et Jean-Claude Fritz • Dominique Gros

Olivier Jouanjan • Catherine Kessedjian

Marie-Claire Laval-Reviglio - Danièle Lochak

Eric Loquin • Jean Marcou • Michael R. Marrus

Gérard Miller - Anne-Françoise Ropert-Précloux

Gérald Simon • Michel Verpeaux • Richard Weisberg

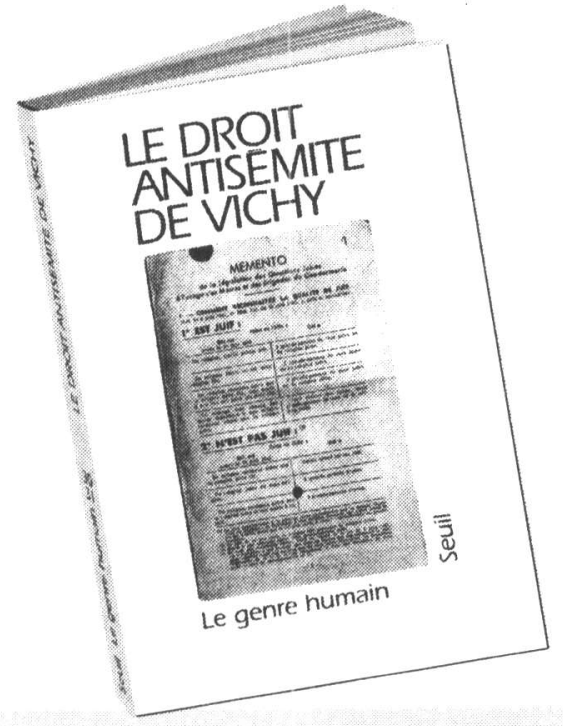

Le genre humain $\mathrm{N}^{\circ} 30-31$

1996

612 pages. $190 \mathrm{~F}$

Annexes, index

et table des sommaires.

ISBN 2-02-029366-2.

RAPPEL :

Le genre humain $\mathrm{N}^{\circ} 28$

Juger sous Vichy

\section{ABONNEMENTS}

Pour 4 numéros : France et DOM-TOM : 370 F - Etranger : $430 \mathrm{~F}$

Le genre humain - Editions du Seuil

(les 25 premiers numéros : $990 \mathrm{~F}$ )

Service des abonnements

BP 29 - 91162 Longiumeau cedex - Tél. 69092490 


\section{IEMOUVEMENT SOCAL \\ AVRIL-JUIN 1996 NUMÉRO 175}

\section{SOMMAIRE}

\section{LA SOCIÉTÉ ET L'ENTREPRISE}

Les liens entre la société et l'entreprise : trois perspectives, par Patrick Fridenson . . . . . . . . . . . . .

La gestion du travail, entre utilitarisme heureux et éthique malheureuse. L'exemple des entreprises françaises au début du XIXe siè-

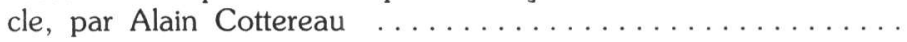

Patronat et autorité patronale : le cas des travaux publics (1883-1974), par Dominique Barjot . . . . . . . . . . . . . . . .

Du veston au bas de soie: identité et évolution du groupe des employés de bureau (1890-1930), par Delphine Gardey ......

Le libéralisme dans l'empire du fer: François de Wendel et la Lorraine industrielle 1900-1914, par David M. Gordon . . . . . . . .

Mouvement social et politiques d'organisation: Peugeot et le pays de Montbéliard de 1919 à 1922, par Yves Cohen ...........

Regards sur la genèse de la pensée nationalisatrice : la nationalisation des banques sous la III e République, par Claire Andrieu ......

Scolarisation et insertion professionnelle des enfants d'ouvriers de Sochaux-Montbéliard, par Stéphane Beaud .............

NOTES DE LECTURE . . . . . . . . . . . . . . .

Michelin, les hommes du pneu, par A. Gueslin et alii (J.M. Laux).

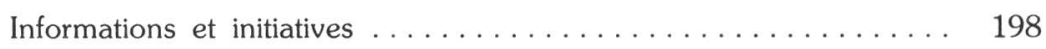

Résumés ............................ 200

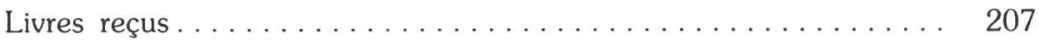

Administration du «Mouvement Social », 12, avenue de la SourRosalie, 75013 Paris.

Abonnement annuel: France et C.E.E. : 220 F, Étranger hors C.E.E. (par avion): $290 \mathrm{~F}$.

Abonnement pour deux ans : France et C.E.E. : $430 \mathrm{~F}$, hors C.E.E. (par avion): $560 \mathrm{~F}$.

Les abonnements étrangers doivent être versés par un mandat international ou chèque libellés en francs français.

VENTE AU NUMERO Le numéro : France et C.E.E. : $70 \mathrm{~F}$, Étranger hors C.E.E. : $82 \mathrm{~F}$. Le "Mouvement Social » est en vente à la librairie des Éditions Ouvrières, 12, avenue de la Sœur-Rosalie, 75013 Paris, ainsi que dans les grandes librairies des villes universitaires.

\section{LES ÉDITIONS DE L'ATELIER}


Revue

française

d'anthropologie

avril

juin

1996
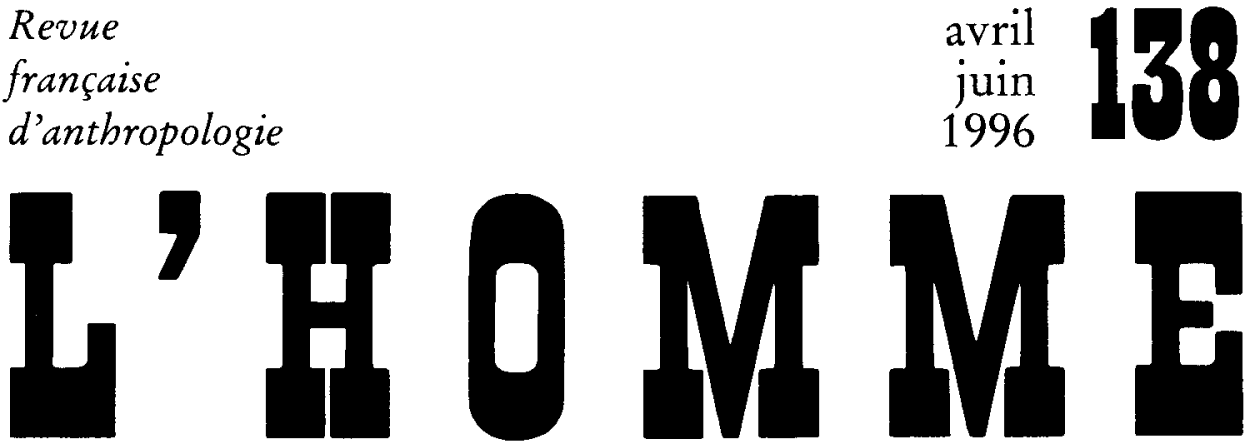

Véronique Boyer

Odile Gannier

Annie Paradis

Thierry Wendling

Patrick Prado

A PROPOS

Emmanuel Terray

Philippe Sagant

Charles-Henry

Pradelles de Latour

Emmanuel Désveaux
Le don et l'initiation

De l'impact de la littérature sur les cultes de possession au Brésil

À la découverte d'Indiens navigateurs

Don Giovanni ou la trajectoire du lièvre

Jouer avec le temps: la pendule des joueurs d'échecs

Paysages sans paysans
L'État contre le pouvoir

De la chasse à l'élevage

Les morts et leurs rites en Afrique

Contrôle des naissances et classes d'âge: un cas limite

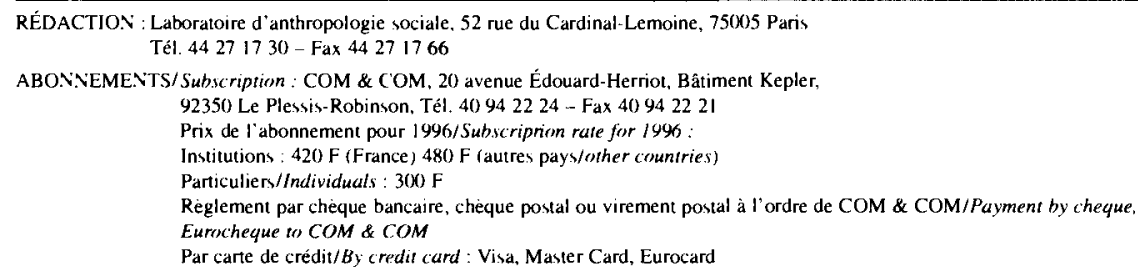




\title{
Sciences Sociales et Santé
}

Vol. $14 n^{\circ} \mathrm{l}$ Mars $1996 \quad$ Revue trimestrielle

\begin{abstract}
La malnutrition de l'enfant : fait culturel, effet de la pauvreté ou du changement social ?
\end{abstract}

\section{Sommaire}

Doris Bonnet Présentation.

La notion de négligence sociale

à propos de la malnutrition de l'enfant

Myriam Roger-Petitjean Représentations populaires

de la malnutrition au Burkina Faso

Yannick Jaffré Dissonances entre les representations sociales et médicales de la malnutrition dans un service de pédiatrie au Niger

\author{
Alice Desclaux Le traitement biomédical \\ de la malnutrition au temps du sida \\ Jean-François Bouville L'approche relationnelle \\ de la malnutrition infantile \\ en milieu tropical
}

La rédaction a reçu

«Sciences Sociales et Santé » est vendu au numéro aux éditions : John Libbey Eurotext

127, avenue de la République, 92120 Montrouge

Tél. (33.1) 46.73.06.60 


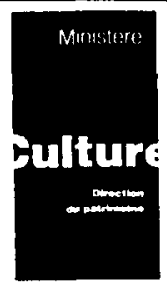

\author{
Mission du patrimoine ethnologique \\ Collection Ethnologie de la France / Regards sur l'Europe \\ CAHIER 10
}

\title{
Sous la direction de Daniel Fabre L'EUROPE ENTRE CULTURES ETNATIONS
}

De la nation moderne, telle que le siècle des Lumières l'engendre, ont été retenus deux actes fondateurs. Le premier ancre l'appartenance dans des références communes, une tradition reconnue. un patrimoine. Le second écarte plus ou moins de ce partage les non-originaires, voire les citoyens de second rang.

Engendrer ses ancêtres et désigner ses étrangers sont les gestes qui inscrivent la nation au coeur de nos sociétés. Pourtant, dans l'Europe du XX $\mathrm{XX}^{\mathfrak{e}}$ siècle, que de différences dans la mise en ceuvre de cette forme commune. Sous l'universalité du modèle national se dessine, dans la façon de traiter du patrimoine ou de l'étranger, une large diversité d'histoires et de cultures.

Aujourd'hui, les bouleversements qui touchent l'Europe ont soudain ravivé un débat dans lequel l'ethnologie se trouve forcément embarquée. Ses mots les plus courants - "culture ", " ethnie ». " tradition», "patrimoine»-ne sont-ils pas parfois devenus des armes?

Venus de six pays, ethnologues et historiens de la culture ont dressé un bilan. ouvert un dialogue. et tracé les perspectives d'un nouveau chantier de l'ethnologie dont ce livre témoigne.

1996, XII-344 p., ISBN 273510719

isSN 07585888 / Collection Ethnologie de la France : ISSN 11600322 / Regards sur l'Europe

Actes du colloque de Tours, décembre 1993 / Textes réunis par Claudie Voisenat et Eva Julien

Gérard Ermisse. Avant-propos

SOMMAIRE

DANIEL FABRE. Introduction

\section{IDENTITÉS ET PATRIMOINES}

Christian Brombergrr. Ethnologie. paurimoine, identités : y a-t-il une spécificité de la situajion française ? Liorenç Prats, Invention de la tradition et construction de l'identité en Catalogne

Josḱ LuIS GARCla, Les biens culturels dans les processus identitaires

PIETRO CLEMENTE. Biens culturels sans culture : le patrimoine ethnologique italien

Marc Maure. Paysan et Viking au musée. Nationalisme et patrimoine en Norvège au xixe siècle

Isac CHIVA, Ethnologie, idéologie et patrimoine

KrZYSZTOF POMIAN. Nation et patrimoine

\section{FIGURES DE L'APPARTENANCE ET LIEN NATIONAL}

DANIEL FABRE. L'ethnologue et les nations

ANDRás ZEMPLÉN1. Les manques de la nation. Sur quelques propriétés de la * patrie " et de la * nation " en Hongrie contemporaine

Harald TAmbS-LyChE, Choisir son pays, choisir son peuple. Remarques sur la genèse norvégienne d'une theorie de l'ethnicité

Pierre Centuivres, A propos des frontières interrieures de la Suisse

Jean-Françols Gosslaux, Un ethnicisme transnational : la résurgence de l'identité valaque dans les Balkans Dolors Comas D' ARGEmir, L'arbre et la maison. Métaphores de l'appartenance

\section{LA PRODUCTION SOCIALE DE L'ÉTRANGER}

GÉrard Althabe, Construction de l'étranger dans la France urbaine d'aujourd'hui VERENA STOLCKE. Europe: nouvelles frontières, nouvelles thétoriques de l'exclusion Gérard et Silvia ARLETTAZ, La a question des étrangers \# en Suisse (1880-1914)

WOLFGANG KaschuBA. Les Allemands, des étrangers les uns pour les autres

Patrick Whalams. Ethnologie, déracinement et patrimoine. A propos de la formation des traits culturels tsiganes

VICTOR KARADY. Les fonctions sociales de l'antisémitisme al l'époque contemporaine. Le cas des États

d'Europe centrale

CIARA GAIIINI, Exotismes de masse

IsAC ChIVA, Conclusion : Les ethnologies de l'Europe, traditions, politiques. perspectives

DıfFusıon, CID, 131, boulevard Saint-Michel F-75005 Paris, Tél. : (1) 43544715 Éditions de la Maison des sciences de l'homme. Paris 


\section{$\infty$ \\ Sociological Research Online}

\section{Visit our website today: http://www.socresonline.org.uk/socresonline}

Edited by Liz Stanley University of Manchester

\section{A New Electronic Journal}

Sociological Research Online is a new international journal which promotes rapid communication among sociologists and is available free of charge on the internet. The first issue was published in March 1996.

The journal publishes fully refereed and high quality, applied sociology, focusing on theoretical, empirical and methodological discussions which engage with current political, cultural and intellectual topics and debates.

\section{Call for Papers}

Sociological Research Online welcomes papers across the entire range of sociological concerns and interests.

If you would like to be kept informed about Sociological Research Online or are interested in submitting a manuscript:

- visit our website <http://www.socresonline.org.uk/socresonline>

- contact Stuart Peters, Department of Sociology, University of Surrey, Guildford GU2 5XH, <socres@soc.surrey.ac.uk>

Sociological Research Online is supported by the Joint Information Systems Committee's Electronic Libraries Programme. It is managed by a consortium of the Universities of Surrey and Stirling, the British Sociological Association and SAGE Publications. 


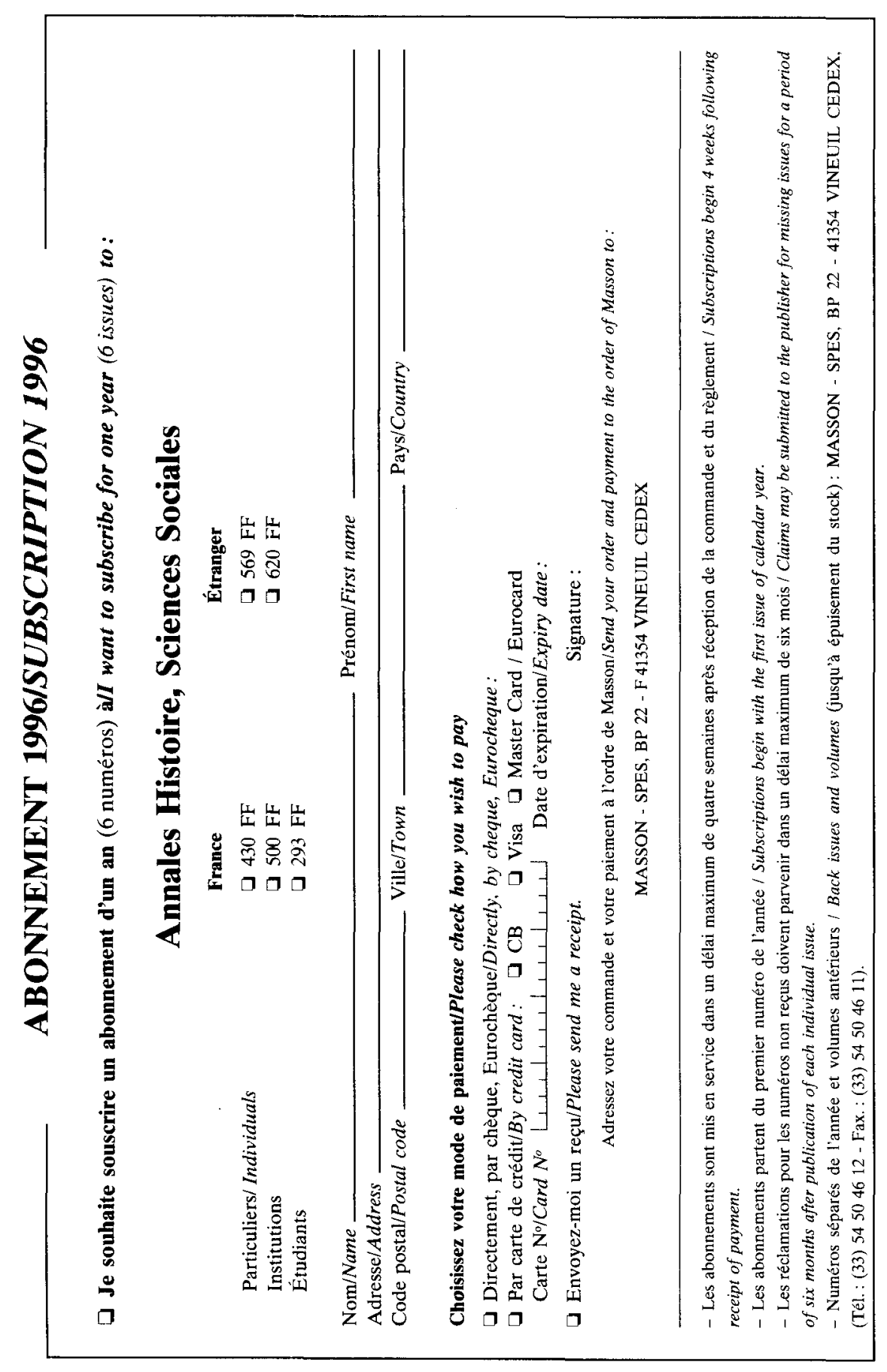




\section{CONGRÈS ET COLLOQUES}

XII• Congrès International d'Histoire Économique.

Prix pour les thèses de doctorat terminées entre le $1^{\text {er }}$ janvier 1993 et le 31 décembre 1996. Les jeunes chercheurs en histoire économique sont invités à présenter des synthèses de leur travail lors du Congrès de l'Association Internationale d'Histoire Économique qui aura lieu à Séville du 24 au 28 août 1998.

Le Comité sélectionnera environ trente finalistes dont les frais d'inscription et de logement à Séville seront pris en charge. Les finalistes pourront aussi demander une subvention pour financer leur voyage. Des résumés de leurs thèses pourront être publiés dans un volume des Actes du Congrès. Tous les finalistes recevront un diplôme et quatre prix de 1500 dollars seront attribués.

- Informations : Pour tout renseignement, s'adresser au professeur Joseph Goy, Secrétaire général de l'AlHE, EHESS-CRH, 54, bd Raspail, 75006 Paris. Fax $n^{\circ}$ 49.54.23.99, e mail : aihe@ehess.fr 


\section{CAHIERS DES ANNALES}

En vente au Comptoir de vente Armand Colin, 12, rue de l'Éperon, 75006 PARIS

M. BLOCH, Seigneurie française et manoir anglais, $2^{\circ}$ édition.

M. AGULHON, Le cercle dans la France bourgeoise (1810-1848).

P. CHAUNU, Histoire quantitative, histoire sérielle.

W. G. L. RANDLES, De la terre plate au globe terrestre.

S. L. KAPLAN, Le complot de famine : histoire d'une rumeur au xvill siècle.

R. HALÉVI, Les loges maçonniques dans la France d'Ancien Régime.

R. E. GIESEY, Cérémonial et puissance souveraine, France, $x V^{0}-x V_{10}$ siècles.

B. MÜLLER, Bibliographie des travaux de Lucien Febvre.

S. ALBERRO, Les Espagnols dans le Mexique colonial.

G. BÉAUR, L'immobilier et la Révolution. Marché de la pierre et mutations urbaines, 1770-1810.

Pour les années 1929 à 1978 ainsi que pour les index analytiques 1929-1948, s'adresser à

KRAUS REPRINT, Route 100, Milwood, New York 10546, USA. 


\section{Lucy, le Jivaro et le supporter \\ Le regard anthropologique}

\begin{tabular}{r|r}
$\begin{array}{r}\text { CONFÉRENCES, DÉBATS, } \\
\text { PROJECTIONS, }\end{array}$ & $\begin{array}{l}\text { au Musée national des arts } \\
\text { et traditions populaires } \\
\text { du } 11 \text { au } 13 \text { octobre } 1996 .\end{array}$ \\
EXPOSITION DE LIVRES &
\end{tabular}

A u-delà des entre les cultures, quels sont les $\mathrm{c}$ a $\mathrm{r}$ a $\mathrm{c} t$ è $\mathrm{r}$ e $\mathrm{s}$ u n i vers a u x conımuns à toutes les sociétés humaines ?

L'anthropologie est la science qui tente de répondre à cette question et c'est autour de cette discipline

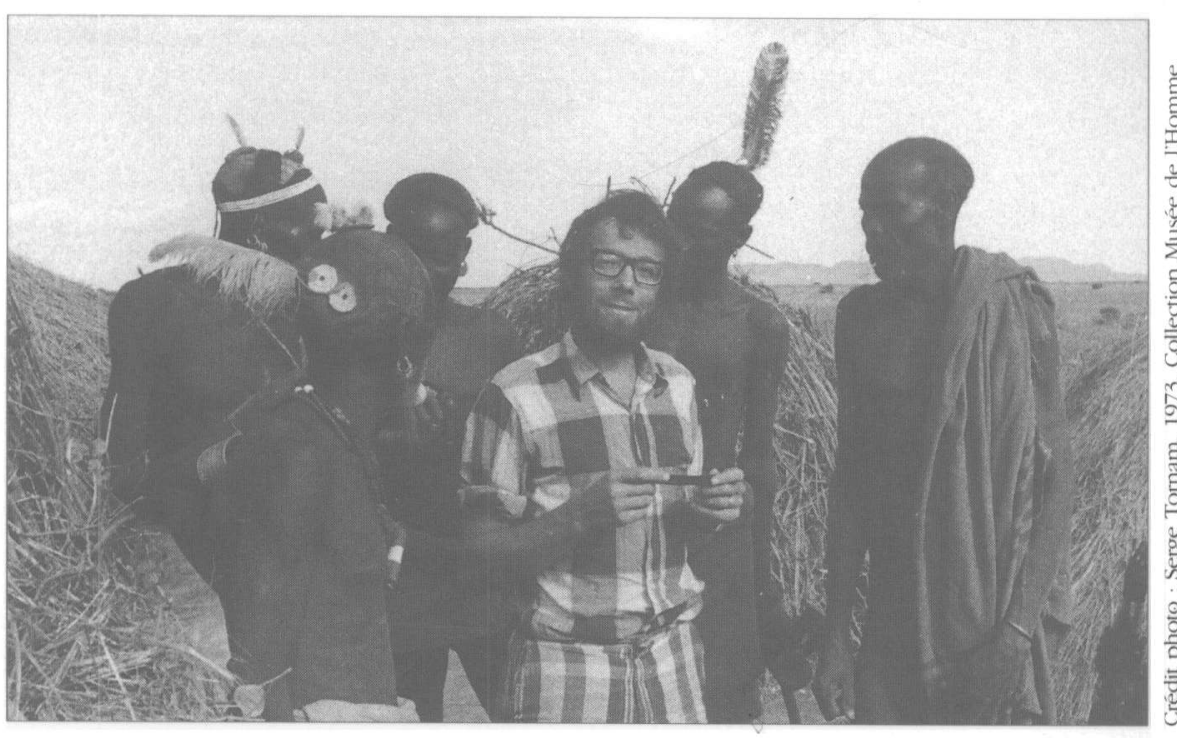
que le Musée national des arts et traditions populaires développe ses collections.

Avec le concours de la Fondation Maison des Sciences de l'Homme et de 흠a Mission du Patrimoine ethnologique, et en partenariat avec les éditions ฏ̀Masson/Armand Colin et CID Diffusion, le Musée vous invite à rencontrer des 跑nthropologues, des ethnologues et des archéologues.

Venez découvrir leurs méthodes et leurs expériences de terrain.

\section{Musée national des arts et traditions populaires}

6 avenue du Mahatma Gandhi - 75116 Paris

Métro : Les Sablons

Information programme : 44176000

Missiom

du Psitrimoine

Etbrologique
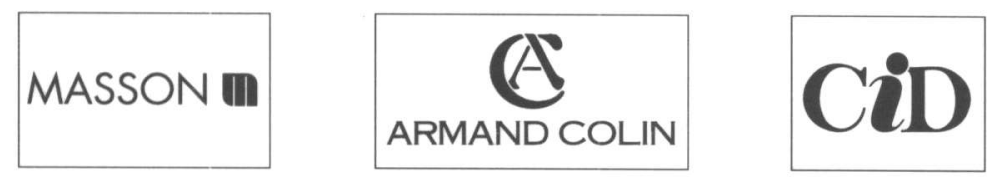

MSH

Paris 